\title{
EFFECT OF REINFORCEMENT ALIGNMENT ON THE PROPERTIES OF POLYMER MATRIX COMPOSITE
}

\author{
M. R. Aeyzarq Muhammad Hadzreel ${ }^{1, a}$ and I. Siti Rabiatull Aisha ${ }^{1, b}$ \\ ${ }^{1}$ Faculty of Mechanical Engineering, University Malaysia Pahang, \\ 26600 Pekan, Pahang Malaysia \\ Email: aaeyzarq89@gmail.com, ${ }^{b}$ rabiatull@ump.edu.my
}

\begin{abstract}
Numerous applications have been proposed and demonstrated for aligned-fiber composites. However, none had stated a correct procedure for aligning the fibers to optimize the properties of the polymer matrix composite (PMC), such as its strength and water absorption properties. Therefore, the aim of this study is to determine the best alignment of reinforcement material in order to optimize the properties of PMC. Woven roving fiberglass was used as the fiber and unsaturated polyester resin as its matrix material. A hand lay-up process was used to fabricate the laminated composite. The specimens were divided into four major categories with different alignments and thicknesses of fiber and matrix, which were five-layer bidirectional, five-layer multidirectional, seven-layer bidirectional, and seven-layer multidirectional. Tensile tests showed that bidirectional alignment offered better mechanical properties compared with the multidirectional alignment. The five-layer bidirectional arrangement has a higher tensile strength compared with five-layer multidirectional arrangement. The seven-layer bidirectional arrangement has higher tensile strength compared with the seven-layer multidirectional arrangement. The modulus of elasticity of the bidirectional alignment was higher than that of the multidirectional alignment. Bidirectional alignment was better because the external tensile load was distributed equally on all the fibers and transmitted along the axes of the fibers. Whereas in the case of multidirectional alignment, the fiber axes were non-parallel to the load axis, resulting in off-axis pulling on the fibers and increased stress concentration, which caused earlier failure of the laminates. Water absorption tests showed that the multidirectional alignment absorbs a greater quantity of water compared with the bidirectional alignment. Therefore, the multidirectional alignment was shown unsuitable for underwater applications, such as boats, canoes, and yachts. It can be concluded that the properties of PMC depend on the alignment of the reinforcement material.
\end{abstract}

Keywords: Reinforcement material; polymer matrix composite.

\section{INTRODUCTION}

Composite materials have become used widely in industries, which include those from the aerospace to the automotive industry and from household products to optical devices, and many other applications (Adebisi, Maleque, \& Rahman, 2011). Composite materials can be defined as at least two elements working together to produce material properties that are different to the properties of each of those elements on their own (Bachtiar, Sapuan, \& Hamdan, 2010; Smith, 2005; Mohamed \& Atan, 2012). The majority of composites are made from a continuous phase called a matrix, in which a stronger phase consisting of fiber or particles is embedded (Umar, Zainudin, \& Sapuan, 
2012; Bhaskar \& Sharief, 2012). Fundamentally, fibers or particles are distributed through a matrix to enhance the properties of the matrix in some way. Polymer matrix composites (PMCs) first emerged as a distinct technology in an era when improved performance for advanced military systems provided the primary motivation for materials development (Ibrahim, Sapuan, \& Faieza, 2012; Kakani, 2006; Mallick 2007; James, Sache, Pielichowski, Leszczynska, \& Giacomelli, 2013). Nowadays, laminated PMC has become used widely in structural applications owing to its high longitudinal modulus and tensile strength, which result from outstanding fiber properties (Perié, Brosse, Girault, \& Leibler, 2011, 2012). Figure 1 shows a laminate fabricated by "cross plying" unidirectional reinforced layers in a $0^{\circ}-90^{\circ}$ stacking sequence. In fact, the most common multidirectional laminates can usually sustain increasing loads after extensive transverse cracking has occurred in various plies, but fiber fracture generally causes overall failure. Table 1 shows some properties of composite reinforcing fibers.

Table 1. Properties of composite reinforcing fibers (Smith, 2005).

\begin{tabular}{lccccccc}
\hline Material & $\begin{array}{c}E \\
(\mathrm{GPa})\end{array}$ & $\begin{array}{l}\sigma \mathrm{b} \\
(\mathrm{GPa})\end{array}$ & $\begin{array}{l}\epsilon \mathrm{b} \\
(\%)\end{array}$ & $\begin{array}{c}\rho \\
\left(\mathrm{Mg} / \mathrm{m}^{3}\right)\end{array}$ & $\begin{array}{c}E / \rho \\
(\mathrm{MJ} / \mathrm{kg})\end{array}$ & $\begin{array}{c}\sigma \mathrm{b} / \rho \\
(\mathrm{MJ} / \mathrm{kg})\end{array}$ & $\begin{array}{c}\text { Cost } \\
(\$ / \mathrm{kg})\end{array}$ \\
\hline E-glass & 72.4 & 2.4 & 2.6 & 2.54 & 28.5 & 0.95 & 1.1 \\
S-glass & 85.5 & 4.5 & 2.0 & 2.49 & 34.3 & 1.8 & $22-33$ \\
aramid & 124 & 3.6 & 2.3 & 1.45 & 86 & 2.5 & $22-33$ \\
boron & 400 & 3.5 & 1.0 & 2.45 & 163 & 1.43 & $330-440$ \\
HS graphite & 253 & 2.5 & 1.1 & 1.80 & 140 & 2.5 & $66-110$ \\
HM graphite & 520 & 2.4 & 0.6 & 1.85 & 281 & 1.3 & $220-660$ \\
\hline
\end{tabular}

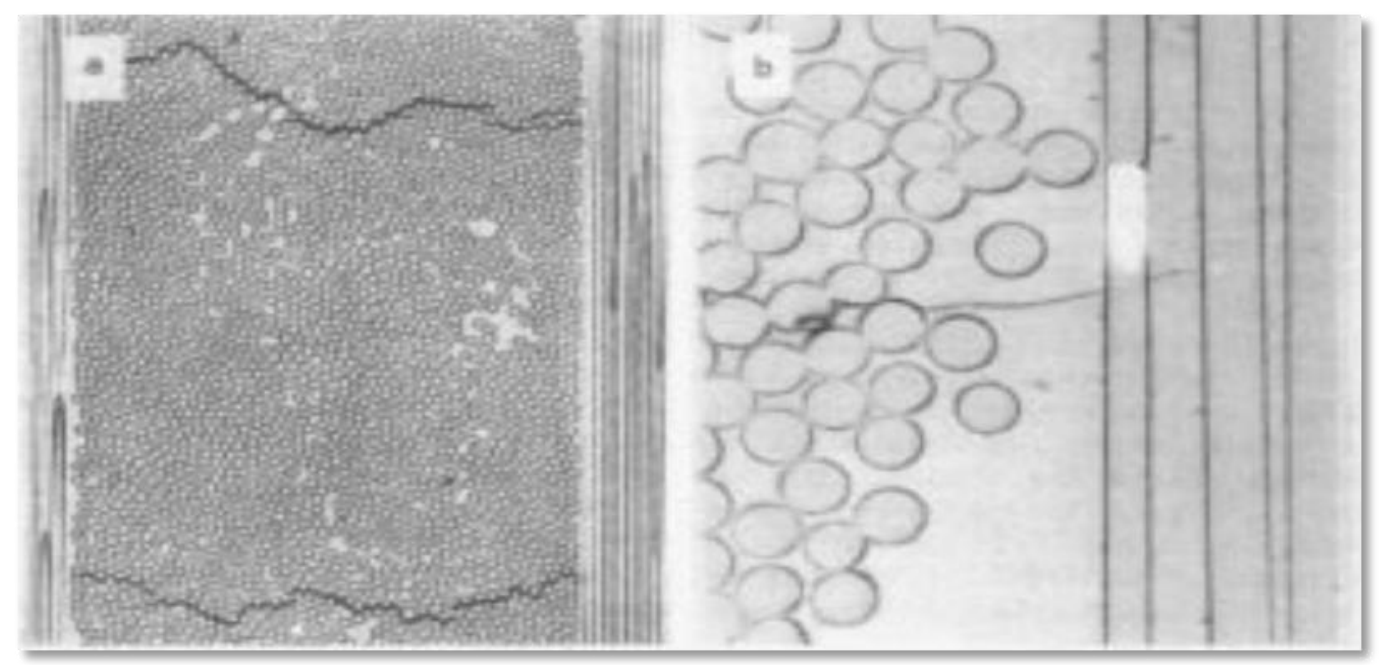

Figure 1. A cross-plied FRP laminate showing non-uniform fiber packing and micro cracking (Harriette, Jorg, \& Martie, 2006).

The aim of this study is to determine the effect of the alignment of the reinforcement material on the properties of PMCs. The properties such as stress, strain, Young's Modulus, and water absorption play important roles in producing the best quality PMC. It is therefore important in terms of both a scientific and a practical point of view, to understand the effects of the alignment of the reinforcement material on the properties of PMCs. 


\section{EXPERIMENTAL PROCEDURES}

This experiment was conducted to distinguish the mechanical and physical properties of PMCs by using different types of alignment and evaluating their water resistance by using different alignments exposed to seawater. As discussed in the previous section, the composite material was a combination of matrix material and reinforcement material. The matrix material used was unsaturated polyester and the reinforcement material was a woven roving fiberglass. There were two types of reinforcement alignment studied in this study, which were bidirectional orientation $\left(0^{\circ}, 90^{\circ}, 0^{\circ}, 90^{\circ} 0^{\circ}\right.$, $\left.90^{\circ}, 0^{\circ}, 90^{\circ}\right)$ and multidirectional orientation $\left(0^{\circ}, 30^{\circ}, 60^{\circ}, 90^{\circ}, 0^{\circ}, 30^{\circ}, 60^{\circ}, 90^{\circ}\right)$. All the specimens were fabricated by using a hand lay-up process because this is the easiest way to fabricate the specimens. Mechanical tests run on the specimens were tensile tests (ASTM D3039) and water absorption tests (ASTM D570).

\section{RESULTS AND DISCUSSION}

\section{Tensile Test}

The tensile test was conducted according to ASTM D3039. This test method determines the in-plane tensile properties of PMC materials reinforced by high-modulus fibers. The composite material forms are limited to continuous fiber or discontinuous fiberreinforced composites, in which the laminate is balanced and symmetric with respect to the test direction. For this work, five specimens were prepared for each thickness and alignment in order to determine the precision of the data. The test was run at a head displacement rate of $2 \mathrm{~mm} / \mathrm{min}$.

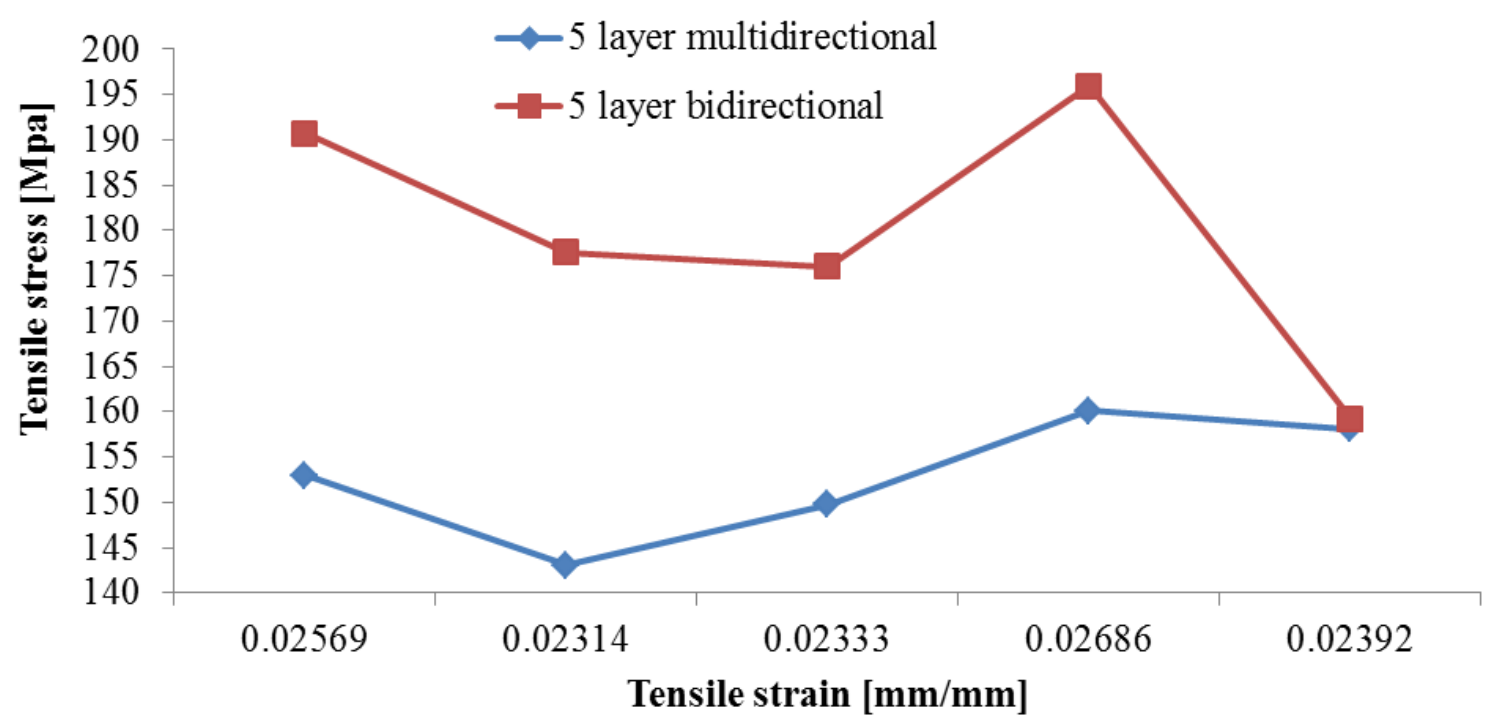

Figure 2. Comparison between ultimate tensile strength for each type of alignment for five-layer thickness.

Figures 2 and 3 show a comparison between each type of alignment of reinforcement material for the five-layer and seven-layer thicknesses. The highest tensile strength for the five-layer bidirectional arrangement is $195 \mathrm{MPa}$, whereas the 
highest tensile strength for the five-layer multidirectional arrangement is $160 \mathrm{MPa}$. The highest tensile strength for the seven-layer bidirectional arrangement is $243 \mathrm{MPa}$, whereas the highest tensile strength for the seven-layer multidirectional arrangement is $219 \mathrm{MPa}$. From the graph, it can be observed that the bidirectional alignment was better in terms of tensile strength compared with the multidirectional alignment. The tensile test graphs showed that the composite material is an elastic material, which means that the material response is instantaneous to the loading. A material is called elastic if the deformation produced in the body is recovered completely following the removal of the load. According to Satish, Siddeshwarappa, and Kaleemull (2010), tensile strengths are affected significantly by fiber orientation. The tensile strength is superior in the case of the $0^{\circ} / 90^{\circ}$ oriented specimens compared with the specimens with $30 / 60^{\circ}$ and $\pm 45^{\circ}$ orientations. This is because the external tensile load is distributed equally on all the fibers and transmitted along the axes of the fibers for the bidirectional alignment. In the case of other fiber orientations, the fiber axes are non-parallel to the load axis, resulting in off-axis pulling of the fibers and increased stress concentration, which causes the earlier failure of the laminates. This study proves that composite material is an anisotropic material, which means that the properties such as ultimate tensile strength and Young's modulus depend on the direction of the loading. A large range of tensile strength values was observed for each type of alignment and thickness because the matrix material was not distributed uniformly on the laminar ply. When the matrix is not distributed uniformly on the laminar ply, the load is concentrated on the reinforcement alone, which causes the failure of the specimen.

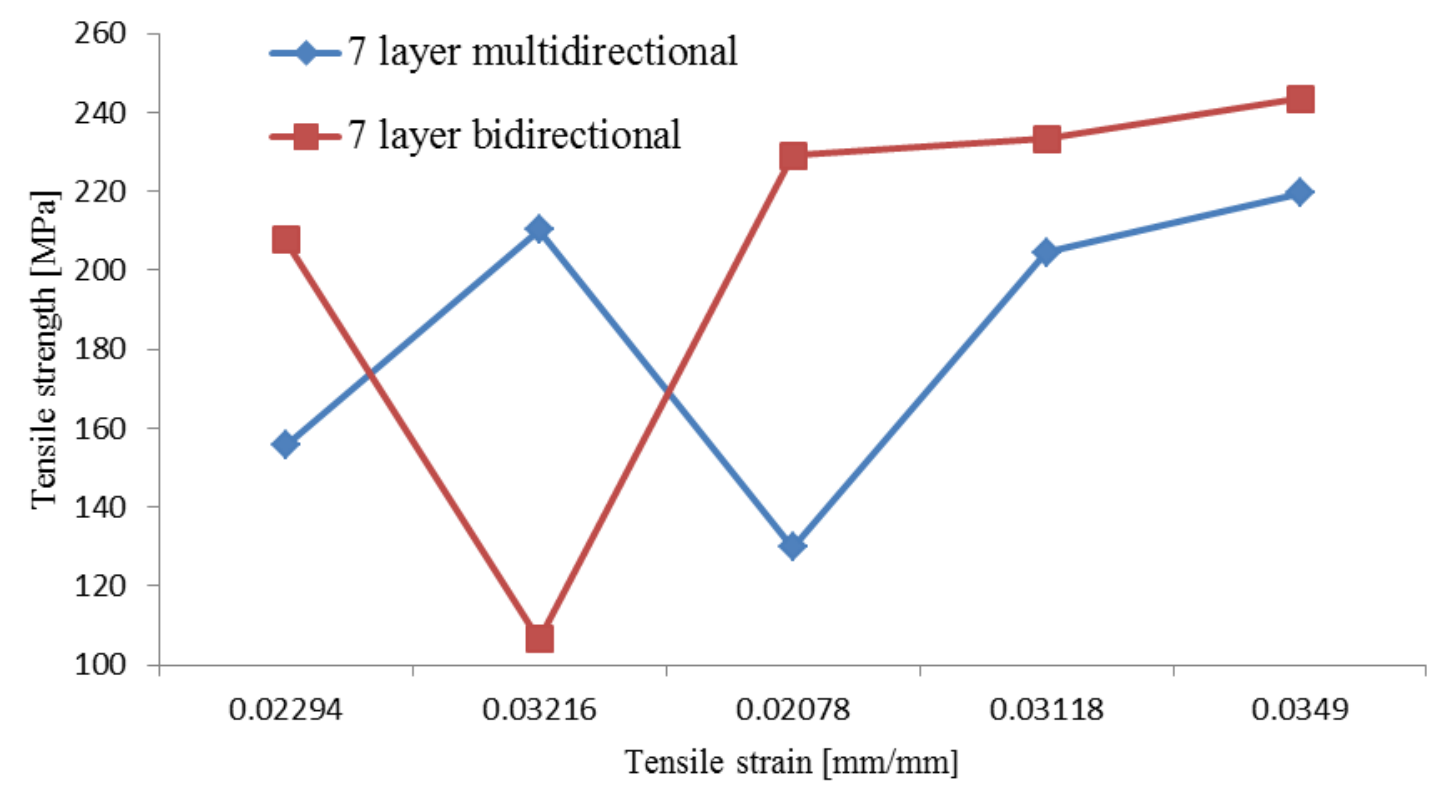

Figure 3. Comparison between ultimate tensile strength for each type of alignment for seven-layer thickness

From Figure 4, we can observe that the Young's modulus was greater for the bidirectional alignment. This is because the properties, such as stress and strain, were greater for the bidirectional alignment. The graph above proves that the bidirectional alignment will make the PMCs stiffer than the multidirectional alignment. Stiffness is the rigidity of an object, which is the extent to which it resists deformation in response 
to an applied force. Harriette et al. (2006) stated that the Young's modulus is directly proportional to the ultimate tensile strength of the material, which means when the ultimate tensile strength increases, the Young's modulus will also increase. For an anisotropic material, the Young's modulus is highly dependent on the direction of loading. The bidirectional alignment revealed a higher Young's modulus because loading was parallel to the axes of the fibers.

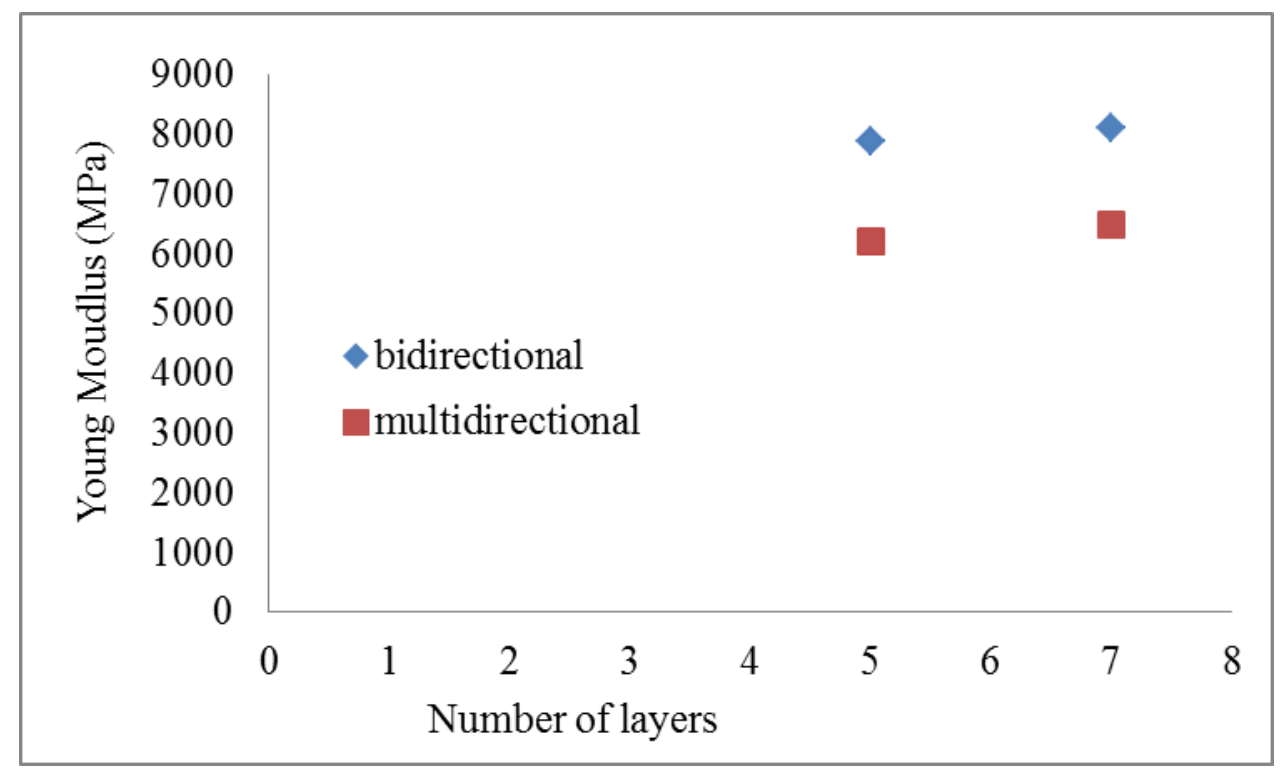

Figure 4. Young's modulus for each type of alignment and thickness.

\section{Water Absorption Test}

Figure 5 shows the percentage of water absorption for each type of alignment and thickness. The seven-layer multidirectional orientation absorbed a greater quantity of water compared with the seven-layer bidirectional orientation. Whereas, the five-layer multidirectional orientation absorbed a greater quantity of water compared with the five-layer bidirectional orientation. Thus, it can be concluded that the multidirectional orientation will absorb a greater quantity of water compared with the bidirectional orientation. Seawater degradation can cause swelling and plasticization of the polyester matrix and debonding at the fiber/matrix interface, which may reduce the mechanical properties. Patel (2008) found that the diffusivity for the specimens in salt water is much higher compared with distilled water and $\mathrm{NaCl}$. They also found that the initial rate of moisture absorption is high. The absorption is Fickian in nature (i.e., it obeys Fick's second law), but gradually, the absorption rate decreases, as is evident from the decreasing slope of the curve. This happens because of saturation of the matrix. The bulky nature of the salts present in seawater ant their osmotic effects were probably the cause of the decrease in the rate of moisture absorption over time. Hence, the bidirectional orientation is more suitable for underwater application, such as yachts, boats, and canoes, because of its resistant characteristic towards seawater. 


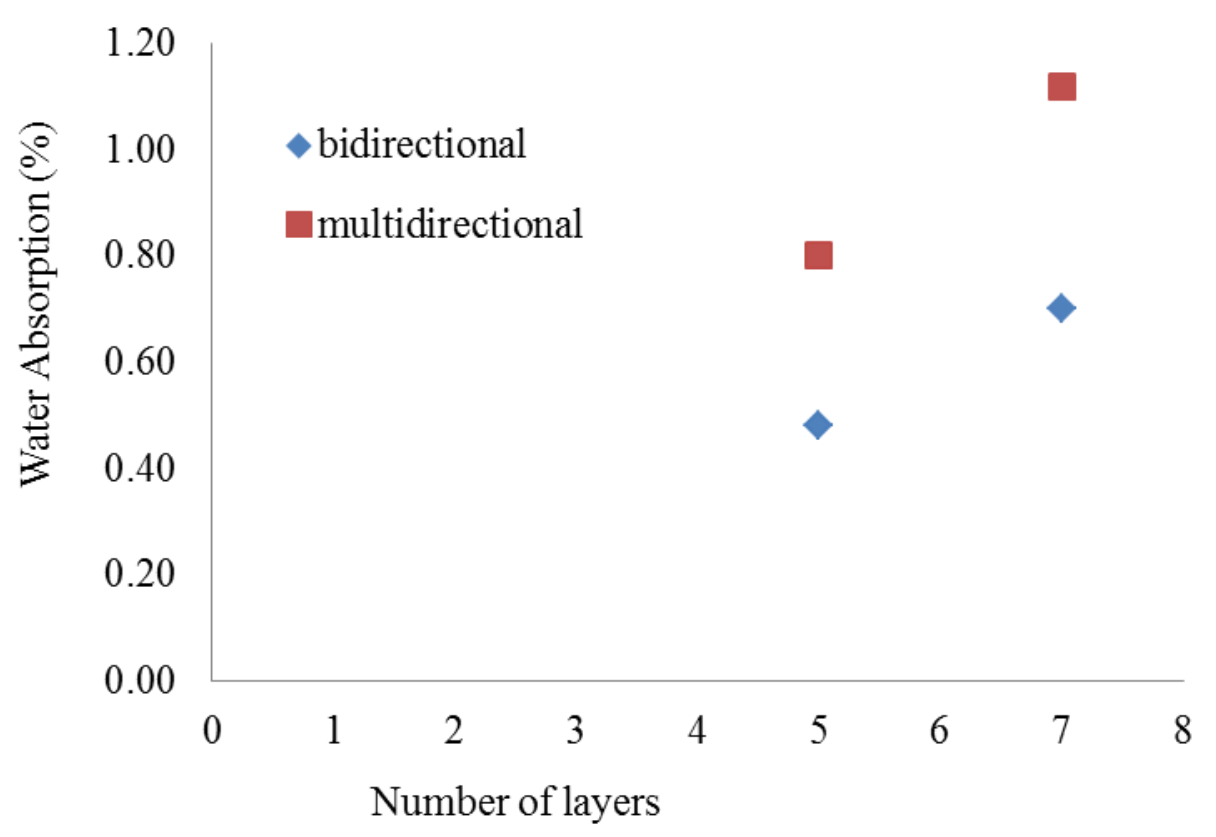

Figure 5. Water absorption test result.

\section{CONCLUSION}

These are the conclusions that can be drawn from the current work:

a. The alignment of reinforcement material affected the properties of the PMC. The bidirectional alignment $\left(0^{\circ}, 90^{\circ}\right)$ offered better mechanical properties, such as ultimate tensile strength and Young's modulus compared with the multidirectional alignment $\left(0^{\circ}, 30^{\circ}, 60^{\circ}, 90^{\circ}, 0^{\circ}, 30^{\circ}, 60^{\circ}, 90^{\circ}\right)$.

b. Water absorption tests showed that the multidirectional alignment absorbed a greater quantity of water compared with the bidirectional alignment. The thickness also affected the water absorption of the PMC. Water absorption was directly proportional to the number of layers of the fiberglass.

\section{ACKNOWLEDGEMENTS}

This work was supported financially by the Universiti Malaysia, Pahang.

\section{REFERENCES}

Adebisi, A. A., Maleque, M. A., \& Rahman, M. M. (2011). Metal matrix composite brake rotor: historical development and product life cycle analysis. International Journal of Automotive and Mechanical Engineering, 4, 471-480.

Bachtiar, D., Sapuan, S. M., \& Hamdan, M. M. (2010). Flexural properties of alkaline treated sugar palm fibre reinforced epoxy composites. International Journal of Automotive and Mechanical Engineering, 1, 79-90.

Bhaskar, H. B., \& Sharief, A. (2012). Effect of solutionizing on dry sliding wear of A12024-Beryl metal matrix composite. Journal of Mechanical Engineering and Sciences, 3, 281-290. 
Harriette, L. B., Jorg, M., \& Martie, J. A. (2006). Mechanical properties of short-flaxfiber reinforced compound. Composite A 37, 1591-1604.

Ibrahim, M. S., Sapuan, S. M., \& Faieza, A. A. (2012). Mechanical and thermal properties of composites from unsaturated polyester filled with oil palm ash. Journal of Mechanical Engineering and Sciences, 2, 133-147.

James, S., Sache, N. S., Pielichowski, K., Leszczynska, A., \& Giacomelli, M. (2013). The influence of multiscale fillers reinforcement into impact resistance and energy absorption properties of polyamide 6 and polypropylene nanocomposite structures. Materials \& Design, 50, 244-252.

Kakani, S. L. (2006). Material Science. New Age International Publishers

Mallick, P. K. (2007). Fiber-Reinforced composites materials, manufacturing, and design. New York: Taylor \& Francis Group.

Mohamed, W. A. N. W., \& Atan, R. (2012). Analysis of excessive heating on the thermal and electrical resistance of a polymer electrolyte membrane fuel cell. International Journal of Automotive and Mechanical Engineering, 5, 648-659.

Patel, D. K. (2008). A comparative study of effects on characteristic properties of FRP composites when exposed to distilled water, nacl- water solution and sea water separately. Bachelor thesis. Department of Metallurgical and Materials Engineering National Institute of Technology, Rourkela, India.

Perié, T., Brosse, A. C., Girault, S. T., \& Leibler, L. (2011). Nanostructured films and composites from carbon nanotubes dispersed by $\mathrm{ABC}$ block terpolymers in selective solvent. Polymer, 52(14), 3065-3073.

Perié, T., Brosse, A. C., Girault, S. T., \& Leibler, L. (2012). Mechanical and electrical properties of multi walled carbon nanotube/ABC block terpolymer composites. Carbon, 50(8), 2918-2928.

Satish, K. G., Siddeshwarappa, B., \& Kaleemull, K. M. (2010). Characterization of inplane mechanical properties of laminated hybrid composites. Journal of Minerals \& Materials Characterization \& Engineering, 9(2), 105-114.

Smith, W. F. (2005). Foundations of materials science. 4th edition. Singapore: McGraw Hill.

Umar, A. H., Zainudin, E. S., \& Sapuan, S. M. (2012). Effect of accelerated weathering on tensile properties of kenaf reinforced high-density polyethylene composites. Journal of Mechanical Engineering and Sciences, 2, 198-205. 